- Qualitative interviews with patients and carers after missed clinic appointments

- Audit (SCD Acute Pain Management Audit) of NICE guidance (CG143)

- Statistical Analysis of Index of Multiple Deprivation (IMD) for all SCD patients, those who missed clinic appointments, and those attending A\&E

Results Semi-structured questionnaire $(n=12)$ :

- $66 \%$ of patients presented to A\&E for urgent treatment rather than GP or home treatment

- $33 \%$ had needed emergency treatment in the last 6 months

- $60 \%$ of patients stated their pain was treated quickly in A\&E (40\% not quickly enough)

- $30 \%$ felt A\&E staff did not know enough about SCD

- Qualitative interviews $(n=5)$ :

- Reasons given for missing appointments included: too many appointments, financial difficulties, travel difficulties, unwell child, appointments booked too far in advance

- Suggestions from families to improve appointment attendance included to send a closer reminder and to follow up telephone confirmations with a letter

SCD Pain Management Audit $(n=28)$ :

- Pain was assessed using an age appropriate scoring tool in $89 \%$ of cases

- We offered analgesia within 30 minutes in only $46 \%$ of cases

- $73 \%$ were prescribed laxatives when on opioids, and $47 \%$ were prescribed anti-emetics or anti-pruritics

Statistical Analysis $(\mathrm{n}=5190)$ :

Preliminary analysis has shown:

- Using the IMD of residence, the most deprived (groups 1-3) had higher rates of missed hospital appointments when compared to groups 4-8

- An increased rate of admission was observed if at least one outpatient appointment was missed

Conclusion Higher rates of missed appointments were observed in the most deprived groups and correlated with increased admissions. Financial difficulties may contribute to missing hospital appointments, adding to health inequities in this vulnerable group.

The next phase will include implementing changes to improve paediatric care and accessibility, including considering virtual or community clinics.

\section{G181(P) PROPHYLACTIC RED CELL AND PLATELET TRANSFUSIONS IN PAEDIATRIC HAEMATO-ONCOLOGY PATIENTS: LESSONS FROM A UK SINGLE-CENTRE RETROSPECTIVE AUDIT}

${ }^{1}$ LLP Hanssen, ${ }^{1} \mathrm{C}$ Hildyard, 'S Staples, ${ }^{1} \mathrm{AJ}$ King, ${ }^{2,3} \mathrm{MF}$ Murphy. 'Paediatric Haematology/ Oncology Unit, Oxford University Hospitals NHS Trust, Oxford, UK; ${ }^{2}$ NHS Blood and Transplant Centre, Oxford University Hospitals NHS Trust, Oxford, UK; ${ }^{3}$ Oxford National Institute for Health Research Biomedical Research Centre, Oxford University Hospitals NHS Trust, Oxford, UK

\subsection{6/archdischild-2020-rcpch. 152}

Aims Transfusion of blood components carries significant risk. Adherence to national guidelines on transfusion thresholds standardises patient care and helps guide decision making. Such guidelines are currently less evidence-based in paediatric than adult practice. This audit aims to capture real-world practice in a tertiary centre paediatric haematology/oncology unit.

Methods Blood transfusions to inpatients over four months between October 2018 and January 2019 were retrospectively analysed. Transfusion details, pre- and post-transfusion haemoglobin and platelet counts, and patient characteristics were obtained from BloodTrack and the electronic patient record (EPR). Patients with haemoglobinopathies were excluded from this audit.

Results Red cell transfusions $(n=121)$ were given to 38 inpatients and prophylactic platelet transfusions $(n=127)$ were given to 21 inpatients. Underlying conditions recorded were ALL $(n=14)$, AML $(n=3)$, Burkitt lymphoma $(n=2)$, aplastic anaemia $(n=1)$, and various solid tumours $(n=18)$.

Red cell transfusions largely conformed to national guidelines, with $95 \%$ given to patients with a pre-transfusion haemoglobin below $80 \mathrm{~g} / \mathrm{l}$ and a median haemoglobin of $72 \mathrm{~g} / \mathrm{l}$. Two units of red cells were given in $12 \%$ of transfusions.

While $34 \%$ of prophylactic platelet transfusions were given to patients with a platelet count $<10.10^{9} / 1,38 \%$ were given to patients with a pre-transfusion platelet count of $20.10^{9} / 1$ or greater without a valid indication documented in EPR. Half of these were given to a single patient with CMV retinitis at risk of retinal haemorrhage. Recorded pre-procedure platelet transfusions were given before lumbar punctures, surgery, lung biopsy, and tunneled central-line insertions. $6 \%$ of these were given at pre-transfusion platelet counts of $>50.10^{9} / 1$, as local guidelines specified a threshold of $<80.10^{9} / 1$ for central line placement.

A small number of patients received the majority of red cell and platelet transfusions; 64\% of red cell transfusion and $71 \%$ of platelet transfusions were given to six and four patients respectively.

Conclusion This single-centre study shows that national guidelines for red cell transfusions are largely followed in paediatric haemato-oncology patients. However, a small number of complex patients and divergent local guidelines were responsible for low adherence to national platelet transfusion guidelines. To improve local transfusion practice, training of junior staff and spread of guidelines within the department are undertaken, after which re-auditing in 12 months is planned.

\section{G182(P) IMPROVING CARE OF FEBRILE NEUTROPENIA PATIENTS: A SPECIALISED PROFORMA AND RISK STRATIFICATION SYSTEM}

HM Yip, N Seeboruth, O Wilkey. Paediatrics, North Middlesex University Hospital, London UK

\subsection{6/archdischild-2020-rcpch.153}

Aims The occurrence of febrile neutropenia $(\mathrm{FN})$ in paediatric oncology patients is a major cause of morbidity and mortality. Focused and timely management of such patients are essential in preventing progression to sepsis. There has been a recent emphasis on risk-based management of $\mathrm{FN}$ patients using prognostic models to stratify them based on risk of severe complications. In line with this emphasis, we are a secondary care centre that developed a specialised FN proforma and risk stratification system. We aimed to evaluate their impact on the care of FN patients in our centre. 